\title{
Advanced Storage Magnetooptical Disk (AS-MO) System
}

\author{
Satoshi Sumi, Akira Takahashi", and Tetsu Watanabe** \\ Hypermedia Research Center, Sanyo Electric Co., Ltd., 180, Ohmori, Anpachi-cho, Anpachi-gun, Gifu 503-0195, Japan \\ *Precision Technology Development Center, Sharp Corporation, 2613-1 Ichinomoto-cho, Tenri, Nara 632, Japan \\ **Giga Byte Laboratories, Sony Corporation, 6-7-35, Kitashinagawa, Shinagawa-ku, Tokyo 141-0001, Japan
}

\begin{abstract}
Advanced Storage MagnetoOptical Disk (AS-MO) is one of the highest density media for removable systems. By employing a read power control method, AS-MO has a capacity of 6 GBytes with wide system margins in a 120 $\mathrm{mm}$ single sided disk, and can be used under various conditions. The disk has many advantages for both $\mathrm{AV}$ and $\mathrm{PC}$ applications.
\end{abstract}

Key words: magnetooptical disk, MSR, MFM, high density recording, read power control

\section{Introduction}

Optical disks such as CD-ROM or magneto-optical disks have become widely used in personal computers and consumer products. CD (Compact Disc) /MD (Mini Disc) has already created a digital audio world. Recently, high density ROM disks such as DVD have made a digital video world. There are big demands for disks of higher speed and larger capacity, particularly in the audio/visual freld. This requires about 7 to 15 times the capacity and about 10 to 20 times the transfer rate of CDs with the same $120 \mathrm{~mm}$ diameter disk. However, there are no rewritable disks with such high performance.

Generally, disk capacity is determined by the resolution of an optical stylus which depends on a laser wavelength $\lambda$ and an objective lens numerical aperture N.A.. For example, the laser beam spot gathering onto the medium is $\lambda / \mathrm{NA}$, so the track pitch limit becomes $\lambda / 4 \mathrm{NA}$. Comparing this limit with the pitch of various media, it has twice the limitation of CDs, and even 1.47 times that of DVD. Therefore, to achieve high recording density within the same optical parameter, it is important to develop $\mathrm{read} / \mathrm{write}$ technologies beyond the optical limitation.

The magnetooptical disk has the technologies to overcome the size limitation of the beam spot while writing or reading. The magnetic field modulation recording method (MFM) with pulsed laser and magnetically induced super-resolution (MSR) disk are the technologies for writing and reading, respectively [1]. There are also technologies to reduce the cross talk between tracks. Land/groove recording provides the wide system margins. Using these technologies, we will be able to make a new optical disk system with high capacity and high performance.

The Advanced Storage MagnetoOptical Disk (AS-MO) is one of the highest density media for removable storage systems. Available technologies for high density $\mathrm{read} / \mathrm{write}$ are integrated into the disk. By improving the track pitch as well as the linear bit density, we have implemented an area density of $5 \mathrm{Gbit} /$ inch $^{2}$. Consequently, AS-MO has the user capacity of 6 GBytes in a $120 \mathrm{~mm}$ diameter single side disk [2] [3].

This report briefly describes the elementary technology of AS-MO, its system and performance.

\section{AS-MO System}

There are many solutions for high-density optical recording. AS-MO adopted available technologies for high density recording. AS-MO technologies are listed in Table 1. For a smaller optical stylus, the optical parameters are the same as for DVD. The $\mathrm{R} / \mathrm{W}$ strategies are laserpumped MFM for writing, and MSR for reading. In consideration of MFM and format efficiency, the modulation code is NRZIpuls with external clocking, and the physical address is a single-side wobbling groove with staggered address. The main parameters of AS-MO are listed in Table 2. User capacity is 6 GBytes with a data transfer rate of 15.3 to $35.6 \mathrm{Mbps}$. The minimum mark length is $0.235 \mu \mathrm{m}$ with a track pitch of $0.6 \mu \mathrm{m}$. The disk has universal format ( $2 \mathrm{~KB} / 32 \mathrm{~KB}$ sector) for $\mathrm{PC}$ and $\mathrm{AV}$ applications.

To reduce laser beam spots and improve the resolution of the optical system, there is a method to expand the N.A. of the objective lens. However, the larger the N.A., the smaller the tilt margin becomes. A thin substrate is needed

Table 1 AS-MO Technologies

LD : $650 \mathrm{~nm}, \mathrm{NA}: 0.6$ (same as DVD)

$120 \mathrm{~mm}$-dia / $0.6 \mathrm{~mm}$-t substrate

Land and Groove Recording (Track pitch : $0.6 \mu \mathrm{m}$ )

MO (for Reliability) \& MSR (CAD)

Laser pumped MFM (for direct Over Write)

PRML (Bit density : $0.235 \mu \mathrm{m}, \mathrm{PR}(1,1)$ or $\mathrm{PR}(1,2,1)$ )

Single sided wobbling address

ZCAV / ZCLV

NRZI+ (DC suppressed code)

External Clocking (Tangential PushPull) 
Table 2 AS-MO parameters

\begin{tabular}{|c|c|}
\hline \multirow{2}{*}{\multicolumn{2}{|c|}{$\begin{array}{l}\text { User Capacity } 6 \mathrm{~GB} \\
\text { Track pitch } \\
0.6 \mu \mathrm{m} \mathrm{Land} / \text { Groove }\end{array}$}} \\
\hline & \\
\hline Min. Mark Length & $0.235 \mu \mathrm{m}$ \\
\hline Disk Diameter & $120 \mathrm{~mm}$ \\
\hline Disk thickness 0. & $6 \mathrm{~mm}$ (1.2 mm for Clamping) \\
\hline Physical address & Staggered Wobbled Groove Address \\
\hline Logical sector size & $2 \mathrm{~KB}$ \\
\hline ECC block size & $2 \mathrm{~KB} / 32 \mathrm{~KB}$ \\
\hline Linear velocity & 4.5 to $10.9 \mathrm{~m} / \mathrm{sec}$ \\
\hline Data transfer rate & 15.3 to $35.9 \mathrm{Mbps}$ \\
\hline W/R strategy $\quad M$ & FM/MSR(CAD) \\
\hline Data Encoding & NRZI plus \\
\hline
\end{tabular}

to solve this problem. As shown in Fig.1, the thickness of AS-MO in the data area is $0.6 \mathrm{~mm}$ in accordance with an N.A. of 0.6 . On the other hand, the thickness of the clamp area is $1.2 \mathrm{~mm}$ for mechanical compatibility with CD or DVD. In order to prevent the tilt of such a thin substrate, a structure with both sides coated has been adopted because of keeping a balance between stresses.

In AS-MO, the write strategy is laser-pumped MFM. In a light modulation recording (LM) system, the smaller the mark, the narrower the write power margin becomes. This is because the top of the temperature distribution is gentle, that is known as pen tip recording. Therefore it is difficult to control the power for recording small marks such as $0.235 \mu \mathrm{m}$ beyond the optical limitation. On the other hand, in the laser-pumped MFM system, the laser beam is pulsed with a recording clock, and the magnetic field modulated with recording data. By overlapping identical shape domains while writing, perfect marks of $0.235 \mu \mathrm{m}$ with minimum jitter can be formed. Furthermore, since the distribution of media temperature is steeper in pulsed laser than that in continuous laser, thermal interference or crosstalk between tracks is also small. The laser-pumped MFM is the method used not only to improve high linear density, but also to promote track pitch of $0.6 \mu \mathrm{m}$. Although the frequency of magnetic field modulation determines a transfer rate, the nominal transfer rate of $\mathrm{AS}-\mathrm{MO}$ is as high as $21.3 \mathrm{Mbps}$ with a laser pulse duty of $30 \%$.

The detection method used to reproduce the signal from

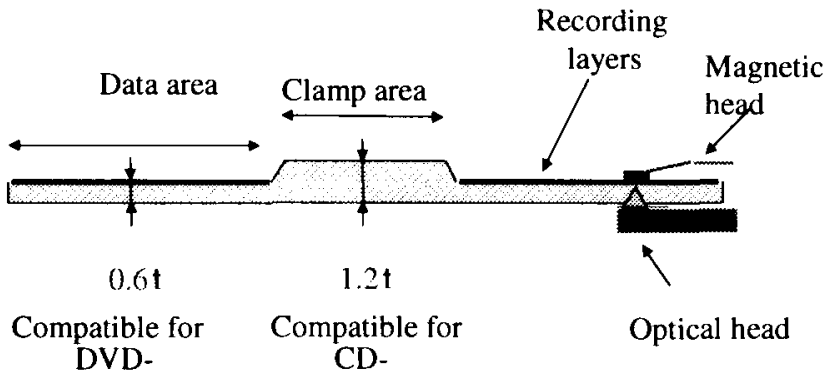

$0.235 \mu \mathrm{m}$ marks is Magnetically induced Super-Resolution (MSR). In the MSR disk, magneto-optical signals are only detected from the aperture area in the laser spot corresponding to the temperature distribution of the disk. AS-MO adapted Center Aperture Detection (CAD) MSR because the disk has good linear properties for write/read and does not need a magnetic field for readout [4]. In CAD-MSR media, the readout layer is in-plane magnetization at room temperature and changes into perpendicular magnetization at high temperatures. Therefore domains in the recording layer are only copied to the high temperature region of the readout layer from the recorded layer. The signals are only reproduced from the center of the laser beam. By using the disk, it is possible to improve the readout resolution, not only in the linear density along track, but also in the track density of the radial direction to suppress the cross talk from adjacent tracks.

Land/groove recording has the advantages of double track density while obtaining almost the same tracking error signals as conventional groove or land recordings. However, since there is no guard band area in land/groove recordings, AS-MO provides an absorbent area for both cross erase and residual marks. As shown in Fig. 2, the track consists of a guard band and an imaginary track. The guard band keeps various system margins such as track servo or write power. The imaginary track is the information track for read/write. In conventional MO media, the track pitch is so narrow that we can not obtain a reproduced signal without cross-talk from adjacent tracks. However, in CAD-MSR media, we can only obtain the signals from an objective track. The cross-talk signals on the adjacent tracks were covered with a CAD-MSR mask. It can be said that the land/groove recording and CADMSR is one of the best combination technologies able to increase track density.

In the AS-MO system, system tolerance is guaranteed to maintain the guard band. Therefore, it is important to control the size and position of the MRS aperture as well as that of the laser beam spot in a conventional system. Generally, the MSR aperture size can change easily with read laser power. AS-MO provides a precise aperture size control that does not destroy this guard band.

Figure 3 shows a block diagram of dynamic read power

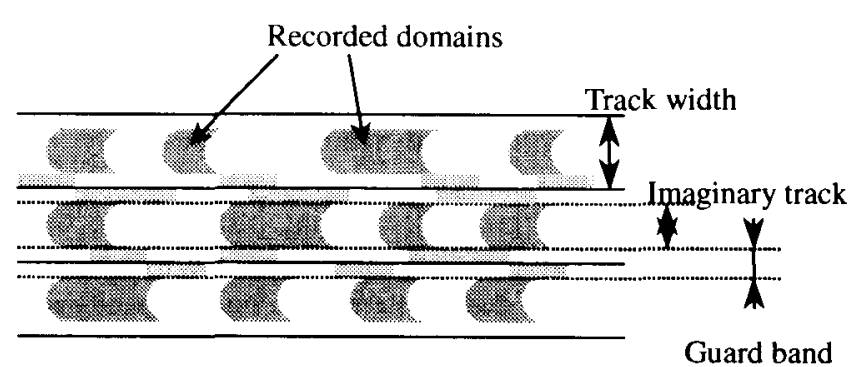




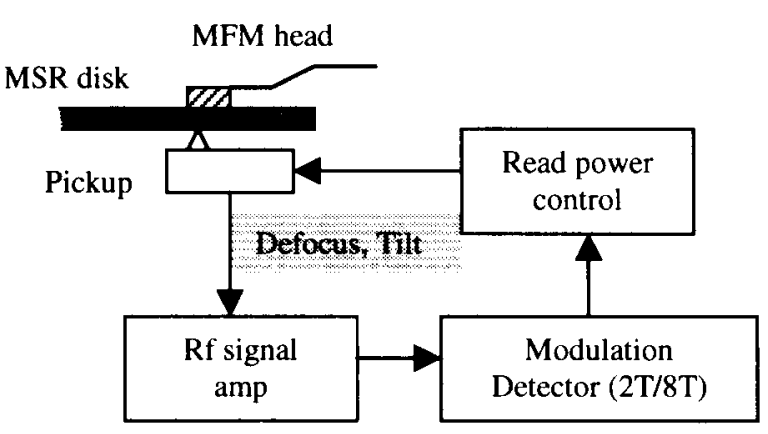

Fig. 3 Dynamic read power compensation

compensation. In this method, the aperture size is detected using the modulation ratio of a $2 \mathrm{~T} / 8 \mathrm{~T}$ signal. The modulation decreases with increasing read laser power because the aperture size is larger in higher read laser power. We can determine the aperture size using a the modulation ratio of $2 \mathrm{~T} / 8 \mathrm{~T}$ [5]. Therefore, the aperture size can be precisely controlled by setting the read laser power to keep the modulation ratio constant. AS-MO has the read power calibration area in every 2 -Kbytes sector, which consist of continuous signals $2 \mathrm{~T}$ and $8 \mathrm{~T}$. An optimum value of the modulation ratio is given by the read power calibration, and a typical value of the ratio is 0.7 to 0.8 in a CAD MSR disk. Using this method, the aperture size will be adjusted to the imaginary track width.

In the case of tilt or defocus, the peek intensity of read laser spot decreases, and the shape of the spot varies. However, in a CAD-MSR disk, signals are reproduced through the readout layer by heating the layer above the threshold temperature, we get good signals by adjusting read laser power. Any irregular beam spots caused by spherical or coma aberration will not affect the quality of the signals because the readout layer masks the cross-talk signals from such irregular spots [6]. By using dynamic read power compensation, the read power can be automatically adjusted in these cases. The dynamic read power compensation is an effective method of not only to putting CAD-MSR to practical use, but also of improving the system tolerance.

\section{Performances}

AS-MO compromised the element technologies as mentioned above. The key technologies in this system are precise control of the MSR aperture size and position, and the write/read laser spot position for removing various external disturbances.

Figure 4 shows the relation between write and read laser power for groove, where the linear bit density is 0.235 $\mu \mathrm{m} / \mathrm{bit}$, the track pitch is $0.6 \mu \mathrm{m}$, and the scanning velocity is $5 \mathrm{~m} / \mathrm{sec}$. A hatched area shows an error rate of less than $1 \times 10^{-4}$. Read power for AS-MO has a bucket curve as in write power, because the aperture size increases with laser power, and both cross-talk and cross-write are suppressed by the MSR mask. The reason for the lower power limit is degradation of SNR. The reason for the higher power limit is the increase of cross-talk from cross-write /residual signals. Therefore, in AS-MO, write laser powers are not independent from read laser powers. Laser power is $\mathbf{1 0 . 5}$ $\mathrm{mW}$ (pulse duty 30\%) for write, and $2.0 \mathrm{~mW}$ for read. Write and read power margin are as wide as $\pm 23 \%$ and $\pm 20 \%$, respectively.

Figure 5 shows the radial tilt read margin with dynamic read power compensation. Disk tilt changes the temperature distribution on the media as a result of changes in the beam profile. Without the read power compensation, the effective read power which generates MSR aperture decreases with increasing disk tilt. As a result, the radial tilt margin without dynamic read power compensation is \pm 13 mrad because of the degradation of SNR. With read power compensation, the system margin is expanded to more than $\pm 21 \mathrm{mrad}$. In an optical disk drive, tilt is one of the most critical parameters. However, the AS-MO system has a wider tilt margin than any

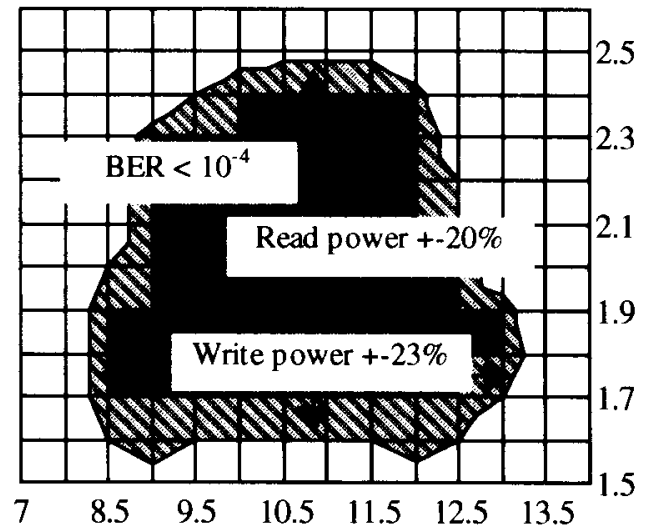

Write power $(\mathrm{mW})$

Fig. 4 Write \& read power margin
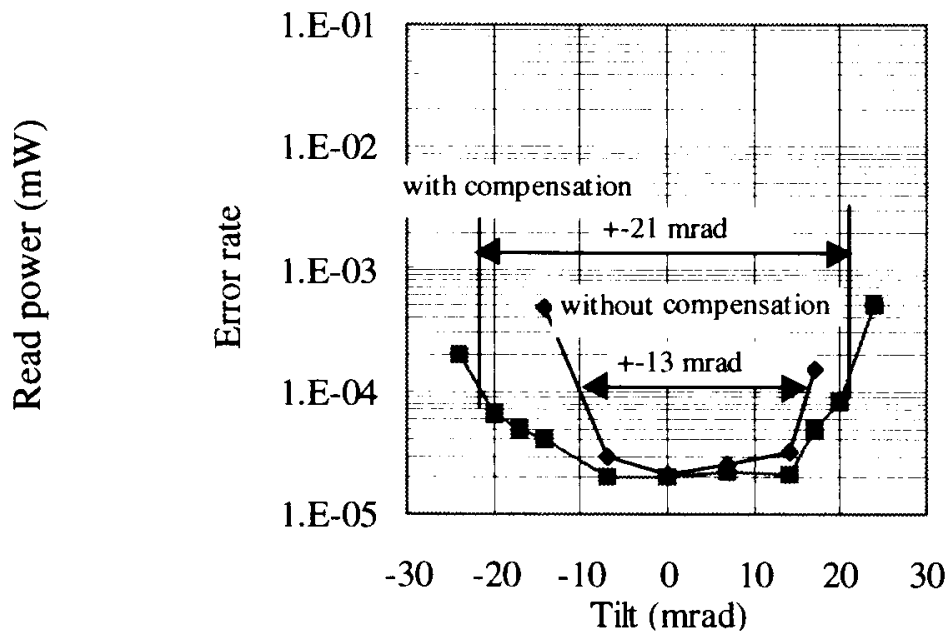

Fig. 5 Radial tilt margin 


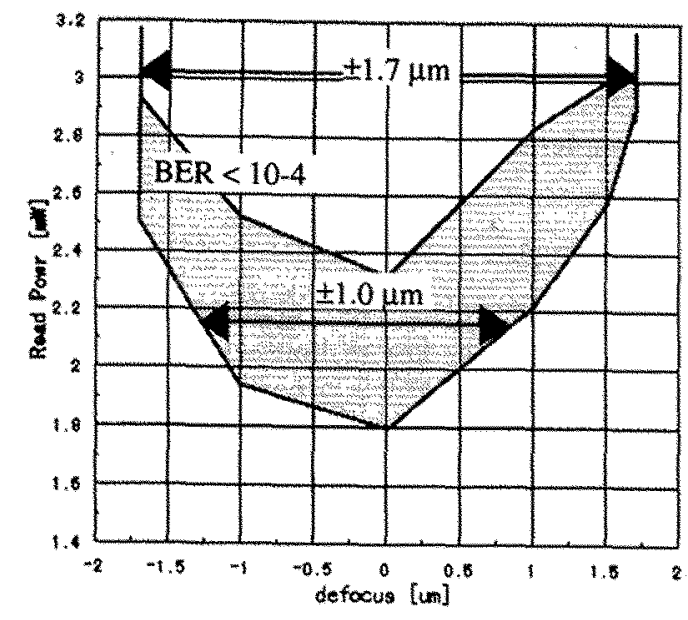

Fig. 6 Defocus margin

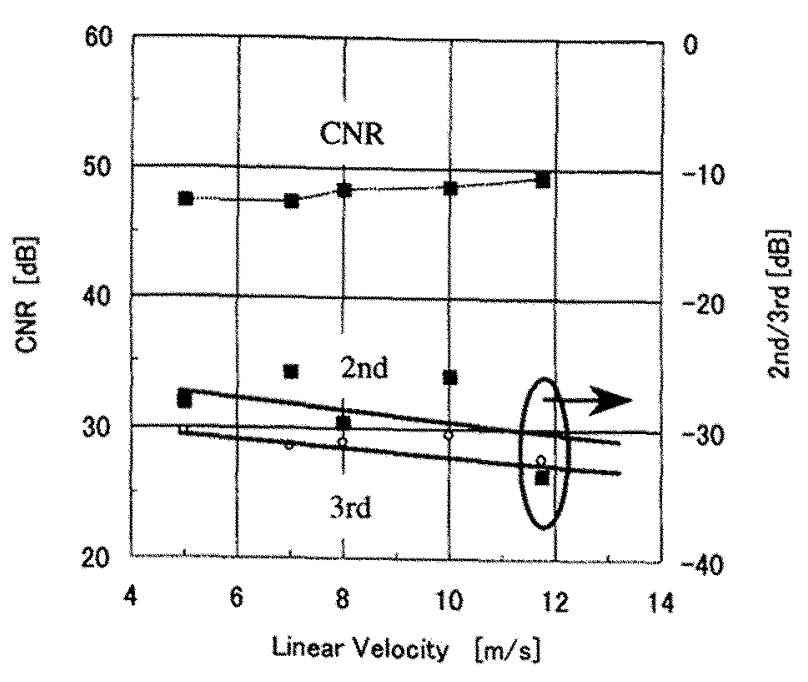

Fig. 7 Distortion of CAD MSR aperture

conventional system.

The defocus margin is shown in Fig. 6. The margin is $\pm 1.0 \mu \mathrm{m}$. However, we can see that the optimum read power moves to higher when defocus is generating. Since the peak intensity of the spot with defocus decreases, the optimum read power increases to generate the same size aperture when it is in focus. By using read power compensation, the defocus margin expands to $\pm 1.7 \mu \mathrm{m}$. In this way, the controlling method for the aperture size has the advantage of enlarging the system margins.

The position of the aperture in a beam spot is also important for signal quality. Figure 7 shows the distortion of the readout signal ( $2 \mathrm{~T}$ ) as a function of linear velocity. The aperture position will be shifted to the rear part of the readout laser spot in a higher linear velocity. However, there are no significant changes to the CNR, or the $2^{\text {nd }}$ and $3^{\text {rd }}$ harmonic signals. The linear velocity of $12 \mathrm{~m} / \mathrm{sec}$ corresponds to a transfer rate of more than $50 \mathrm{Mbps}$. The CAD-MSR aperture is stable for various perturbations.

\section{Conclusion}

AS-MO has allowed writing methods to record domains beyond the optical limitation by using laser-pumped MFM. Furthermore, CAD-MSR media and land/groove recording have improved the track density as well as the linear density. The read power controlling methods have also allowed detection of the steady reproduced signal under various optical deviations. As a result, AS-MO became able to provide enough margins to allow the design of a drive system. The disk has a capacity as high as 6 GBytes in a $120 \mathrm{~mm}$ diameter single sided disk. The capacity is more than 2.3 times that of DVD-RAM, and even 1.27 times that of DVD-ROM.

Future methods of improving capacity will have higher disk capacity and a smaller mark. This will lead to a decreased reproduced signal. The small signal will limit the data transfer rate as well as the recording bit density. It

will be necessary to compensate reproduced signal for a higher data transfer rate or a larger capacity than that of AS-MO. A magnetic domain expansion readout such as MAMMOS is one of these technologies [7]. It is expected that there will be more progress on both high density and high data transfer rate technologies. For example, by using a blue laser diode, the capacity of AS-MO will be easily improved to 15 GBytes by simply covering the optical parameter.

AS-MO has high performance with large system margins. The disk will have many applications for both PC and $\mathrm{AV}$.

Acknowledgement The authors would like to thank the members of ASTC (advanced storage technical conference) for cooperating with measurements and their helpful discussions.

\section{References}

[1] Y. Tanaka, S. Sumi, N. Matsubayashi, H. Sato, H. Awano, M. Matsuura, G. Fujita and T. Watanabe: Jpn. J. Appl. Phys., Vol. 37, 2150 (1998).

[2] A. Takahashi, M. Kaneko, H. Watanabe, Y. Uchihara and M. Moribe : J. Magn. Soc. Jpn., Vol. 22, No. \$2, 67 (1998).

[3] K. Torazawa, S. Sumi, S. Yonezawa, N. Suzuki, Y. Tanaka, A. Takahashi, Y. Murakami and N. Ohta : IEICE Transactions on Electronics, Vol. E80-C, No. 9, 1025 (1997).

[4] J. Hirokane and A. Takahashi : Jpn. J. Appl. Phys., Vol. 35, 5701 (1996).

[5] H. Fuji, Y. Murakami, H. Sato and S, Terashima: J. Magn. Soc. Jpn., Vol.19, No.S1, 441 (1995).

[6] S. Murata, S. Hori, Y. Suzuki and S. Sumi : Opt. Rev, Vol. 5, No. 6, 345 (1998).

[7] H. Awano, A. Yamaguchi, S. Sumi, S. Ohnuki, H. Shirai, N.Ohta and K.Torazawa, : Appl. Phys. Lett., Vol. 69, No. 27, 4257 (1996). 